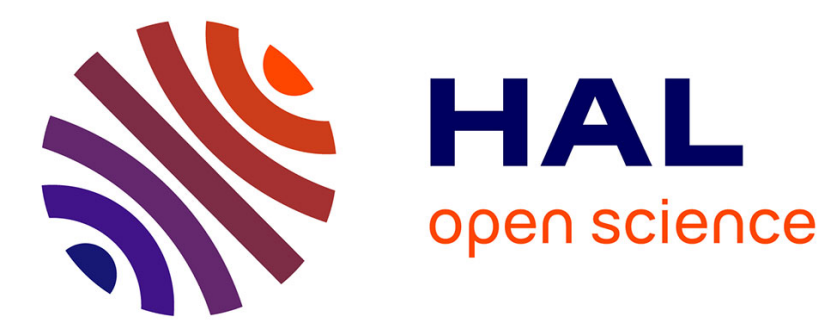

\title{
Open-Thru de-embedding for Graphene RF devices
}

Giancarlo Vincenzi, Georgios Deligeorgis, Fabio Coccetti, Patrick Pons

\section{To cite this version:}

Giancarlo Vincenzi, Georgios Deligeorgis, Fabio Coccetti, Patrick Pons. Open-Thru de-embedding for Graphene RF devices. International Microwave Symposium (IMS), Jun 2014, Tampa Bay, Florida, United States. pp.Proceedings IMS. hal-01024248

\section{HAL Id: hal-01024248 \\ https://hal.science/hal-01024248}

Submitted on 15 Jul 2014

HAL is a multi-disciplinary open access archive for the deposit and dissemination of scientific research documents, whether they are published or not. The documents may come from teaching and research institutions in France or abroad, or from public or private research centers.
L'archive ouverte pluridisciplinaire HAL, est destinée au dépôt et à la diffusion de documents scientifiques de niveau recherche, publiés ou non, émanant des établissements d'enseignement et de recherche français ou étrangers, des laboratoires publics ou privés. 


\title{
Open-Thru de-embedding for Graphene RF devices
}

\author{
Giancarlo Vincenzi ${ }^{* \dagger}$, George Deligeorgis ${ }^{* \dagger}$, Fabio Coccetti ${ }^{* \dagger}$ and Patrick Pons* ${ }^{* \dagger}$ \\ ${ }^{*}$ CNRS, LAAS, 7 avenue du colonel Roche, F-31400 Toulouse, France \\ ${ }^{\dagger}$ Univ de Toulouse, LAAS, F-31400 Toulouse, France
}

\begin{abstract}
The performances of graphene RF devices rely heavily on the precision of the de-embedding techniques adopted, and the small size of the lines increase variability issues in the RF standards and test devices. In this work, a robust and high precision de-embedding technique adapted to the needs of microwave graphene devices is presented. Two standards and only one step after SOLT calibration are needed.
\end{abstract}

Index Terms-Calibration, frequency measurements, graphene, integrated circuit modeling, thin films.

\section{INTRODUCTION}

After nine years from its isolation, graphene is emerging as a valuable candidate in the fields of High-Frequency electronics, as active element, and Flexible and Transparent electronics, as interconnect material [1]. In both fields the high parasitic impedances of graphene/metal contact and interconnects are a critical issue, which greatly limits extrinsic transistor Figures of Merit (FoMs) as $f_{T}$ and $f_{\max }$. De-embedding allows to correct those numbers and boosts intrinsic FOMs up to the double [2], a dramatic improvement compared to III$\mathrm{V}$ semiconductor devices. The error in de-embeddeding can lead to an error in intrinsic FoMs of the same order of the improvement. A precise and robust de-embedding procedure is critical in graphene RF electronics research.

Multiline Thru-Reflect-Line (TRL) [3] is often regarded as the most accurate calibration algorithm as it does not require the fabrication of high-precision resistors. Neverthless, its validity at lower gigahertz frequencies is limited by the need of impractically long reference lines, especially if integrated in low- $\kappa$ substrates. Another widely used de-embedding algorithm is the Open-Short (OS) [4]. It performs very well at low frequency, but being based on a lumped element circuit model, it requires the lengths of the pads and signal-to-ground interconnects to be smaller than $\lambda / 20$. For this reason, it should be used after both SOLT calibration and TRL de-embedding, for a total of three steps [5]. Thru-only algorithms decompose the Thru in $\pi$-shaped or T-shaped [6] networks, but these methods are frequency-limited in the same way as the OS is.

The study of novel materials for RF as metallic nanowires [7], CNT [8] and graphene [9] is performed from DC to many tens of $\mathrm{GHz}$. Moreover, the impedance to be measured ranges from hundreds of $\Omega$ to many $\mathrm{k} \Omega$, offering a poor match to $50 \Omega \mathrm{RF}$ instrumentation and with significant variations in impedance from device to device. The calibration is done up to the probe tips on a standard calibration substrate, while the de-embedding of the interconnects and pads is done through the TRL [7], through the OS [8], or even neglected [9]. In the case of active devices, the OS technique is generally adopted [2].

In this work, the Open-Thru (OT), a broadband deembedding procedure adapted to the characterization of the high-frequency impedance of graphene and thin films is introduced. Its use allows for a more precise extraction of the contact and surface impedance of graphene, together with a more precise extraction of active device small-signal parameters and FOMs. In Section II the method will be detailed; in Section III the de-embedding will be tested on a few graphene passive interconnects and compared with the widely adopted OS; in Section IV a high-frequency model of graphene will be extracted; and in Section V the robustness of this method will be measured.

\section{METHOD}

The OT is an extension of the Thru-only method presented in [10], where the matrix related to each access fixture is first derived by the manipulation of the S-parameters of the Thru, then converted into ABCD chain parameters. In this work a different formulation of the Thru-only is detailed, where the matrix is derived already in the ABCD domain. The final deembedding of the data is the same for the two formulations.

\section{A. Thru-Only de-embedding}

In the Thru-Only method, the uncorrected DUT is thought as the chain of a left fixture, the DUT to be de-embedded, and the right fixture. The ABCD parameters of the Thru standard are square-rooted, inverted and left- and right-multiplied to the ABCD parameters of the DUT, as clearly described in (1).

$$
\mathbf{D}_{\mathbf{T}}=(\sqrt{\mathbf{T}})^{-1} \mathbf{D}_{\text {raw }}(\sqrt{\mathbf{T}})^{-1}
$$

where $\mathbf{D}_{\text {raw }}$ are the measured ABCD parameters of the DUT, $\mathbf{T}$ those of the Thru standard and $\mathbf{D}_{\mathbf{T}}$ are the de-embedded data. The square-root $\mathbf{R}$ of a matrix $\mathbf{M}=\left(\begin{array}{ll}a & b \\ c & d\end{array}\right)$ is instead computed as follows:

$$
\mathbf{R}=\frac{1}{t}\left(\begin{array}{cc}
a+s & b \\
c & d+s
\end{array}\right)
$$

with

$$
s= \pm \sqrt{\delta}
$$

and

$$
t= \pm \sqrt{\tau-2 s},
$$

and $\tau$ and $\delta$ are respectively the trace and the determinant of M. Two solutions exist for the square root of the matrix of a generic delay line (two more exist and are identical to the first pair). However, only one of them is a physical solution, the 
TABLE I

PARAMETERS OF THE CIRCUIT IN FIg. 1.

\begin{tabular}{lccccc}
\hline & TL $Z_{0}$ & TL length & $C_{S}$ & $C_{P 1}$ & $C_{P 2}$ \\
\hline Central value & $47.5 \Omega$ & $300 \mu \mathrm{m}$ & $50 \mathrm{fF}$ & $5 \mathrm{fF}$ & $5 \mathrm{fF}$ \\
Dispersion & $\pm 2.5 \%$ & $\pm 5 \mu \mathrm{m}$ & $\pm 5 \%$ & $\pm 5 \%$ & $\pm 5 \%$ \\
\hline & $R_{S 1}$ & $R_{S 2}$ & $R_{P}$ & $L_{P}$ & \\
\hline Central value & $1 \Omega$ & $1 \Omega$ & $0.5 \Omega$ & $1 \mathrm{pH}$ & \\
Dispersion & $\pm 5 \%$ & $\pm 5 \%$ & $\pm 5 \%$ & $\pm 5 \%$ & \\
\hline
\end{tabular}

other one gives anti-symmetric delay in forward and reverse transmission $\left(<S_{21}\right.$ and $<S_{12}$ are rotated of $\pi$ in Smith chart representation).

A general requirement of the Thru-only method is that the Thru device has to be reciprocal and symmetrical, which is not always the case because of some small and yet unavoidable calibration errors after the SOLT step and/or probe landing misplacement. This requirement in S-parameter notation translates in a bi-symmetrical matrix, i.e. simultaneously symmetrical around the main diagonal $\left(S_{21}=S_{12}\right.$, reciprocal device) and the secondary one $\left(S_{11}=S_{22}\right.$, symmetrical device). However, every matrix can easily be separated in a symmetrical and anti-symmetrical component with simple average and difference operations. The small imperfections left by the SOLT calibration can be subtracted away and incorporated in the de-embedding error, and the modified Thru reference structure can be assumed as the chain of its square roots. Hence, the T matrix in (1) must be the ABCD transform of the bi-symmetrized S-parameters of the Thru.

\section{B. Open-Thru de-embedding}

The Thru-only can be expanded to include the Open standard. Both the DUT and the Open standard are first corrected with the Thru-only method, then the Y-matrix of the corrected Open is subtracted from the DUT's one, as expressed by (5):

$$
\mathbf{D}_{\mathrm{de}-\mathrm{emb}}=\mathbf{D}_{\mathbf{T}}-\mathbf{O}_{\mathbf{T}},
$$

where $\mathbf{D}_{\mathbf{T}}$ and $\mathbf{O}_{\mathbf{T}}$ are the Y-matrixes respectively of the DUT and Open corrected with (1) in ABCD domain and $\mathbf{D}_{\mathbf{d e}-\mathbf{e m b}}$ is the final de-embedded data.

\section{SimULATION AND COMPARISON}

The test device is a circuital model drawn in ADS Schematic. The DUT to be corrected is shown in Fig. 1. It contains the intrinsic section DUT $_{i n t}$, two $300 \mu \mathrm{m}$ slightly mismatched lossy transmission lines at the inputs, and parasitic components $C_{S}, C_{P 1}, C_{P 2}, R_{S 1}, R_{S 2}, R_{P}$ and $L_{P}$. The central values of the capacitors are taken from the model of a series discontinuity as described in $\S 9.5$ of [11], and the inductor and resistances from [5].

The Short and the Open standards are built according to the specifications in [12], and the Thru contains all the elements with the exception of the short-to-ground impedance.

The de-embedding method presented here will be applied to the measurements of a graphene-loaded CPW line of reduced dimension fabricated on a flexible Polyimide (PI) substrate. The Material Under Test (MUT) is placed in series connection

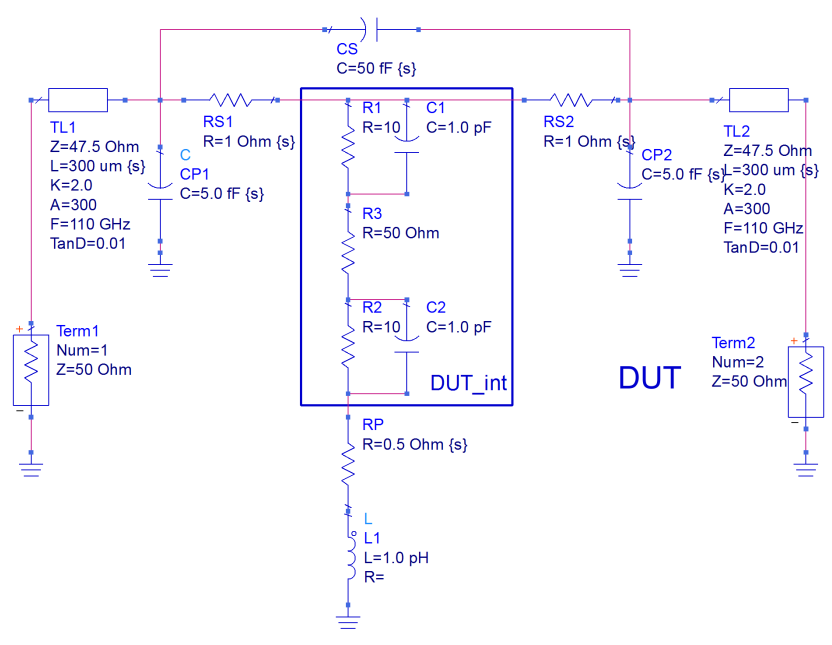

Fig. 1. The circuital model of the extrinsic DUT. In the middle, framed, is the intrinsic part of the device that must be de-embedded.

between the two signal lines. The CPW ground-to-ground width has been chosen of $25 \mu \mathrm{m}$ to maintain the compatibility with in-lab mechanically exfoliated graphene. This results in line gaps of $1.5 \mu \mathrm{m}$ for $50 \Omega$, very close to the resolution limit of the lithographic process used $(1 \mu \mathrm{m})$ and yielding a variation of $\pm 0.13 \mu \mathrm{m}$, measured with low-field SEM. This results in a variation in the $Z_{0}$ of the lines of the $2.4 \%$ from nominal value and up to the $4.8 \%$ of the capacitance between CPW signal and ground electrodes.

A study of the robustness of the de-embedding method against geometrical variations can be easily performed introducing a 5\% Gaussian dispersion on circuit parameters, in all standards and uncorrected DUT, and simulated with a MonteCarlo analysis. The central values and the relative dispersions are detailed in Table I. The resulting S-parameters are shown in Figures 2 and 3. The output variation of each method is comprised between the symbols (triangles and circles) of the respective color. The errors of OS and OT techniques are listed in Table II, averaged between 0 and $110 \mathrm{GHz}$. They are evaluated as $\left|\operatorname{err}\left(\left|S_{11}\right|_{d B}\right)\right|+\left|\operatorname{err}\left(\left|S_{21}\right|_{d B}\right)\right|$ for magnitudes and $\left|\operatorname{err}\left(S_{11 \text { deg }}\right)\right|+\left|\operatorname{err}\left(S_{21 \text { deg }}\right)\right|$ for phases. The OS is more accurate at low frequency because it includes the short-toground impedance, but at $18 \mathrm{GHz}$ the interconnects are $\lambda / 20$ and the error grows quickly. The OT performs better than OS at $8.5 \mathrm{GHz}$ and higher frequencies. The error spread in phases is better for OS and is three times better for OT in magnitudes.

An additional study reducing the interconnect length to $30 \pm$ $5 \mu \mathrm{m}$ has been carried out. The results are in Table II, showing again better performance of OT for magnitudes and of OS for phases. In this case OT is more accurate than OS after $\sim 60 \mathrm{GHz}$.

\section{Graphene Interconnects Measurements}

Graphene-loaded CPW lines have been fabricated. CVD monolayer graphene provided by Graphene Supermarket ${ }^{\circledR}$ has been deposited on pre-fabricated Au CPW lines included 


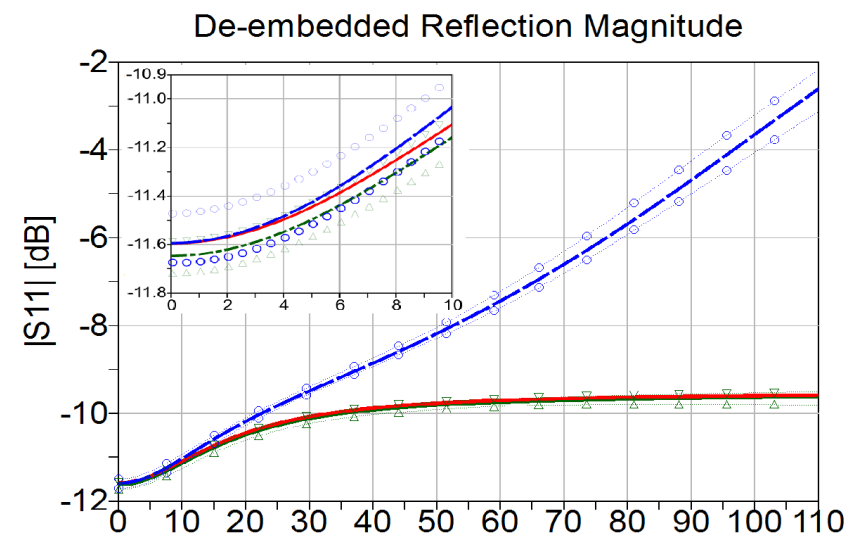

Fig. 2. Simulations of the Reflection magnitude. Comparison between Open-Short (blue dashed line and blue circles), Open-Thru (green dot-dashed line and triangles) and the target DUT $_{i n t}$ (solid red line). Inset: zoom at $0-10 \mathrm{GHz}$.

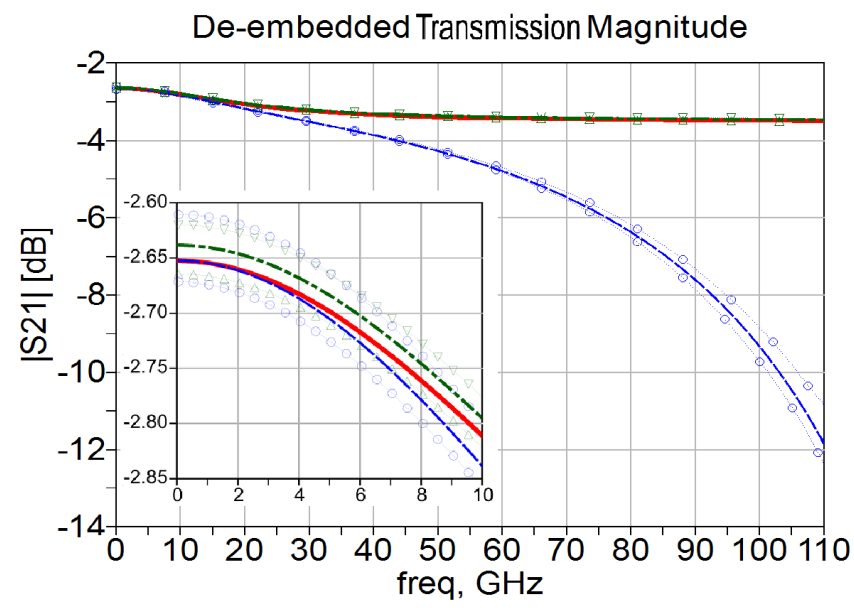

Fig. 3. Same as Fig. 2, simulation of the the Transmission magnitude.

in a PI flexible substrate, shown in Fig. 4, and then patterned in strips of $20 \mu \mathrm{m}$ of width, shown in Fig. 5. The electrode separation is $2 \mu \mathrm{m}$ for the first device and $20 \mu \mathrm{m}$ for the second. The two graphene strips are electrically short at all tested frequencies. The lines have been measured with an Agilent PNA-X up to $67 \mathrm{GHz}$ calibrated at $-20 \mathrm{dBm}$ of power, so that graphene is in linear regime [8]. The fabricated access lines are longer than $180 \mu \mathrm{m}$ on each side, so the OT method has been used. One possible model for graphene at RF frequencies is the lumped element circuit in Fig.6. It is composed by the sheet resistance $R_{s h}$, the contact resistance $R_{C}$ and the contact capacitance $C_{C} . R_{C}$ originates from the cascade of transport of electrons in graphene under the metal and their tunneling into the metal [13]. $C_{C}$ is mainly used to model the charge accumulation at the interface in case of non-ideal contact. However, in the case of graphene, a fraction of $C_{C}$ is predicted to be originated from its intrinsic physics [13]. Hence, $C_{C}$ and $R_{C}$ should be incorporated in graphene's behavior.

The sum of the resistors in Fig. 6 is fixed to the DC resistance $R_{d c}$, measured with a multimeter. Fig. 7 shows the
TABLE II

OPEN-SHORT AND OPEN-THRU DE-EMBEDDING ERRORS.

\begin{tabular}{llcc}
\hline Line length & Method & Magnitude [dB] & Phase [deg] \\
\hline \multirow{2}{*}{$300 \mu \mathrm{m}$} & Open-Short & $4.49 \pm 0.25$ & $2.28 \pm 2.23$ \\
& Open-Thru & $0.08 \pm 0.07$ & $1.26 \pm 2.83$ \\
\hline \multirow{2}{*}{$30 \mu \mathrm{m}$} & Open-Short & $0.13 \pm 0.07$ & $1.50 \pm 1.56$ \\
& Open-Thru & $0.08 \pm 0.06$ & $1.84 \pm 3.06$ \\
\hline
\end{tabular}
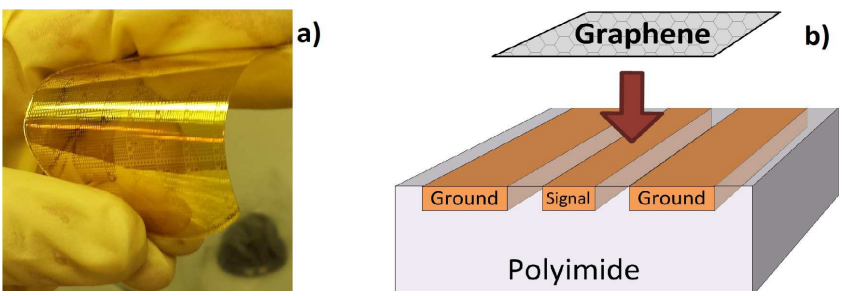

Fig. 4. The flexible PI substrate with included CPW lines (a), and the deposition of graphene on the same (b).

de-embedded data and simulations, shows excellent agreement on all frequencies as in DC, proving that the fixture resistance has been correctly removed. Table III resumes the extracted parameters. $R_{s h}$ and $C_{C}$ differences are consistent with measured $R_{d c}$ and can be explained with non-ideal contacts.

TABLE III

EXTRACTED CONTACT AND SHEET RESISTANCES .

\begin{tabular}{ccccc}
\hline Length & $R_{d c}[\Omega]$ & $R_{s h}[\Omega]$ & $R_{C}[\Omega \cdot \mathrm{mm}]$ & $C_{C}[\mathrm{pF} / \mathrm{mm}]$ \\
\hline $2 \mu \mathrm{m}$ & 240 & 1021 & 1.64 & 6.40 \\
$20 \mu \mathrm{m}$ & 421 & 251 & 2.02 & 0.78 \\
\hline
\end{tabular}

\section{RoBUSTNESS}

The $2 \mu \mathrm{m}$ graphene device has been de-embedded also with the OS method in order to evaluate the sensitivity to non-ideal standards. The OT and OS algorithms both use the

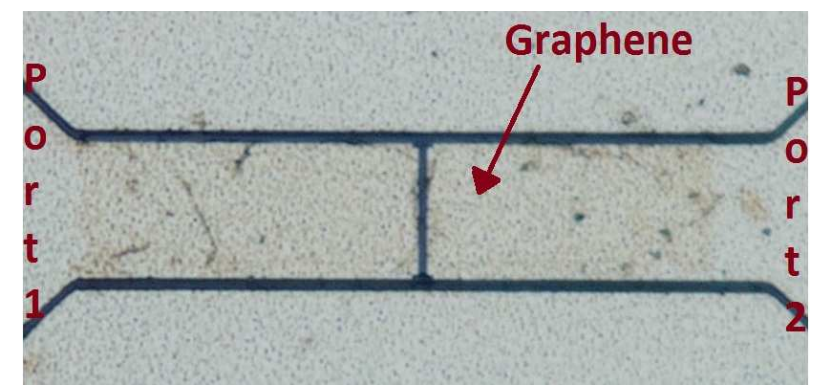

Fig. 5. A $20 \mu$ m-wide graphene strip deposited on the signal line of the CPW on plastic substrate.

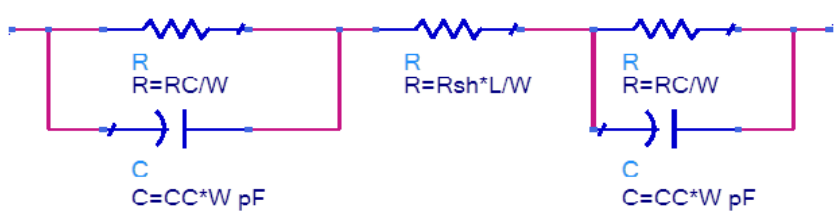

Fig. 6. Graphene RF model. 


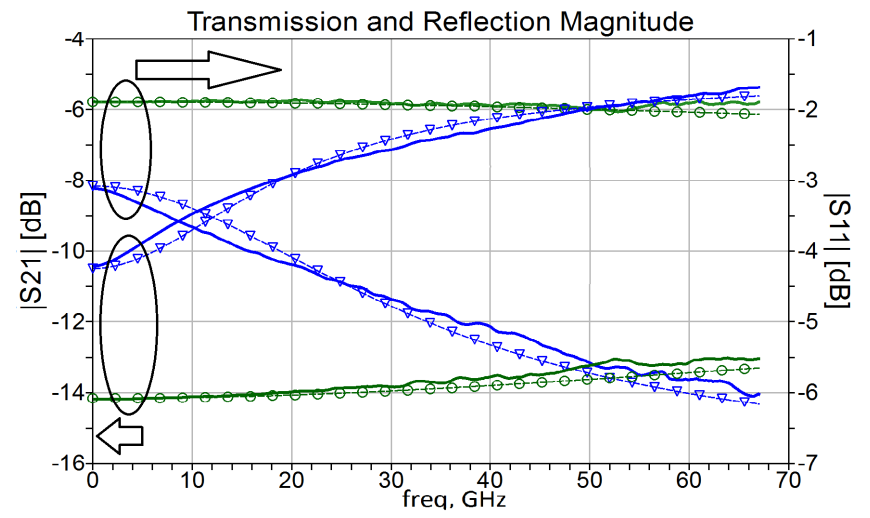

Fig. 7. De-embedded and simulated S-parameters for two graphene devices of $2 \mu \mathrm{m}$ (blue triangles) and $20 \mu \mathrm{m}$ (green circles) of length. Transmission magnitude on left axis, reflection on the right.

same Open standard, of which various copies on the same sample have been measured. The de-embedded data have been averaged over all Open standards, and the errors associated to standard variation have been compared between the two methods. This is shown for the transmission magnitudes in Fig. 8 and for transmission phases in Fig. 9. In both cases the OT method resulted in smaller error due to imperfections in standards compared to OS, i.e. 36\% smaller for magnitudes and $20 \%$ smaller for phases, averaged over all frequencies. The increased robustness of this algorithm might reduce the error associated to the DUT modeling or, conversely, the number of reiterated measurements necessary for the same accuracy. This would increase the standard devices' life and the time employed in measurements campaigns.

The accuracy of this method can be further enhanced by also taking into account the Short standard, making it an improved Open-Short-Thru de-embedding protocol, which would allow the treatment of devices with a low impedance path to ground. However, depending on the impedance of the DUT and on to the resemblance of the electrostatics between DUT and standards terminations, it's not trivial to determine if all the standards in such OST method should be employed. A dedicated study would be necessary to further explore those topics.

In conclusion, the Open-Thru method has been proven as more accurate than the Open-Short for long interconnects, and more robust against imperfections in standards, both in Monte-Carlo simulations and in measurements of micrometric graphene strips. Non-ideal standards are frequently encountered in case of graphene and other carbonaceous thin films RF devices, whose small dimensions are close to lithographic reproducibility limits. The high-precision de-embedding method allowed for accurate modeling of the graphene interconnects under test.

\section{REFERENCES}

[1] K. S. Novoselov et al.,"A roadmap for graphene", Nature, vol. 490, no. 7419, pp. 192200, Oct. 2012.

[2] Z. Guo et al., "Record maximum oscillation frequency in C-face epitaxial graphene transistors", Nano Lett., vol. 13, no. 3, pp. 9427, Mar. 2013.

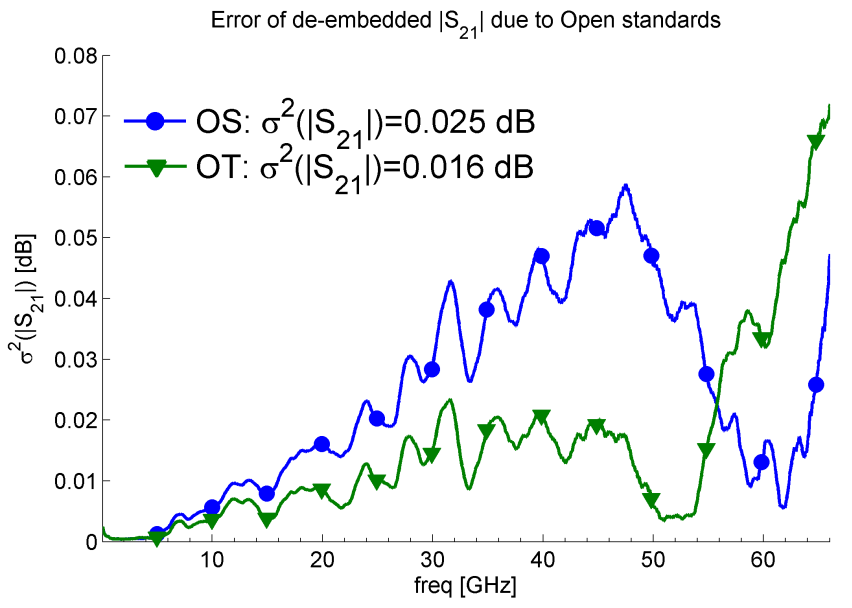

Fig. 8. Error of the transmission magnitude evaluated for the Open-Short (blue circles) and Open-Thru (green triangles) for the measured graphene $2 \mu \mathrm{m}$ strip, expressed as the variance of the set of corrected transmission magnitudes in $\mathrm{dB}$. In the inset, the error averaged over all frequencies for Open-Short and Open-Thru.

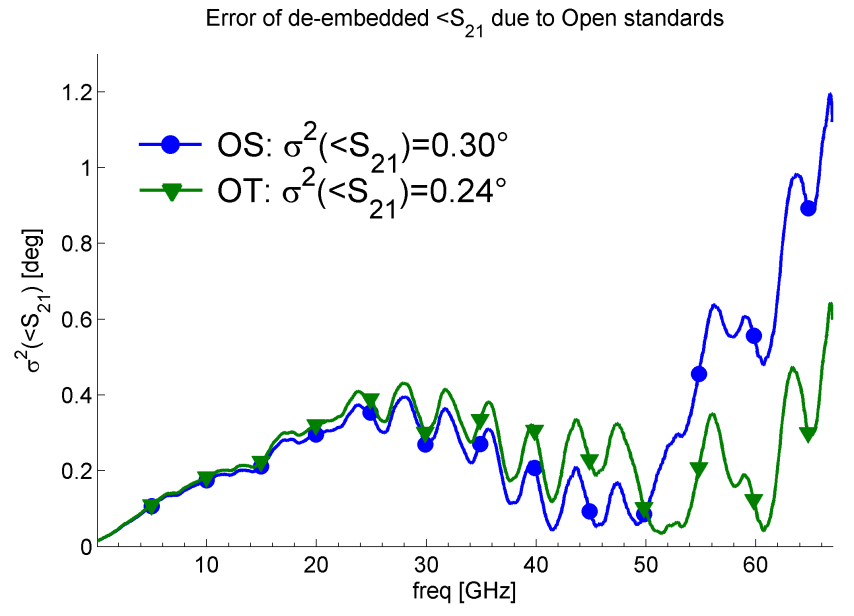

Fig. 9. As above, for transmission phases.

[3] R. B. Marks, "A multiline method of network analyzer calibration", IEEE Trans. Microw. Theory Tech., vol. 39, no. 7, pp. 12051215, Jul. 1991.

[4] M. C. A. M. Koolen, J. A. M. Geelen, and M. P. J. G. Versleijen, "An improved de-embedding technique for on-wafer high-frequency characterization", in Proc. of the 1991 Bipol. Circ. and Tech. Meet., 1991, pp. 188191.

[5] K. Yau et al., "Device and IC Characterization Above $100 \mathrm{GHz}$ ", IEEE Microw. Mag., vol. 13, no. 1, pp. 3054, Jan. 2012.

[6] M. J. Kobrinsky et al., "Experimental validation of crosstalk simulations for on-chip interconnects using S-parameters", IEEE Trans. Adv. Packag., vol. 28, no. 1, pp. 5762, Feb. 2005.

[7] K. Kim et al., "Contactless Approaches for RF Characterization of Metallic Nanowires”, in Microw. Conf. (EuMC), 2010 European, 2010, no. September, pp. 691694.

[8] A. Tselev et al., "Microwave impedance spectroscopy of dense carbon nanotube bundles", Nano Lett., vol. 8, no. 1, pp. 1526, Jan. 2008.

[9] H. S. Skulason et al., " $110 \mathrm{GHz}$ measurement of large-area graphene integrated in low-loss microwave structures", Appl. Phys. Lett., vol. 99, no. 15 , p. $153504,2011$.

[10] T. E. Kolding, "On-wafer calibration techniques for giga-hertz CMOS measurements", in ICMTS 1999. Proc. of 1999 Intern. Conf. on Microel. Test Struct., 1999, no. March, pp. 105110.

[11] R. N. Simons, "Coplanar Waveguide Circuits, Components, and Sys- 
tems". Wiley-IEEE Press, 2001, p. 464.

[12] Y. Cheng, M. Deen, and C. Chen, "MOSFET Modeling for RF IC Design", IEEE Trans. Electron Devices, vol. 52, no. 7, pp. 12861303, Jul. 2005.

[13] F. Xia et al., "The origins and limits of metal-graphene junction resistance", Nature nanotech., vol. 6, no. 3, pp. 17984, Mar. 2011. 